MATHEMATICS OF COMPUTATION

Volume 72, Number 244, Pages 1597-1610

S 0025-5718(02)01488-6

Article electronically published on December 3, 2002

\title{
MAXIMUM-NORM ESTIMATES FOR RESOLVENTS OF ELLIPTIC FINITE ELEMENT OPERATORS
}

\author{
NIKOLAI YU. BAKAEV, VIDAR THOMÉE, AND LARS B. WAHLBIN
}

\begin{abstract}
Let $\Omega$ be a convex domain with smooth boundary in $R^{d}$. It has been shown recently that the semigroup generated by the discrete Laplacian for quasi-uniform families of piecewise linear finite element spaces on $\Omega$ is analytic with respect to the maximum-norm, uniformly in the mesh-width. This implies a resolvent estimate of standard form in the maximum-norm outside some sector in the right halfplane, and conversely. Here we show directly that such a resolvent estimate holds outside any sector around the positive real axis, with arbitrarily small angle. This is useful in the study of fully discrete approximations based on $A(\theta)$-stable rational functions, with $\theta$ small.
\end{abstract}

\section{INTRODUCTION}

Resolvent estimates for elliptic partial differential operators may be utilized in the study, via semigroup theory, of the corresponding parabolic equations, and this holds also for spatially semidiscrete approximations of such equations, e.g., by finite element methods. Estimates of the type needed may often be obtained easily in the $L_{2}$ norm, whereas estimates in $L_{p}$ for $p \neq 2$ are more difficult to derive; this paper is devoted to resolvent estimates in the maximum-norm for the discrete Laplacian associated with piecewise linear finite element spaces.

Let $\Omega$ be a bounded domain with smooth boundary in $d$-dimensional Euclidean space $R^{d}$, and consider the positive definite operator $A=-\Delta=-\sum_{j=1}^{d} \partial^{2} u / \partial x_{j}^{2}$ with homogeneous Dirichlet boundary conditions. Let $\mathcal{C}=\mathcal{C}(\bar{\Omega})$ be the Banach space of continuous complex-valued functions on $\bar{\Omega}$, with norm $|v|=\sup _{x \in \Omega}|v(s)|$, and let $\mathcal{C}_{0}=\{v \in \mathcal{C} ; v=0$ on $\partial \Omega\}$. A special case of a result by Stewart [26] (see Theorem 2.1 below) shows that for any $\varphi \in\left(0, \frac{1}{2} \pi\right)$ there is a constant $C$ such that, if $D(A)=\mathcal{C}^{2}(\bar{\Omega}) \cap \mathcal{C}_{0}$, then

(1.1) $\quad\left|(\lambda I-A)^{-1} v\right| \leq C(1+|\lambda|)^{-1}|v|, \quad$ for $\lambda \notin \Sigma_{\varphi}=\{\lambda ;|\arg \lambda| \leq \varphi\}, v \in \mathcal{C}$.

If we were to consider $A$ as an operator in $\mathcal{C}$, it would not be densely defined, and the standard results of semigroup theory therefore would not automatically apply

Received by the editor September 7, 2001 and, in revised form, March 1, 2002.

2000 Mathematics Subject Classification. Primary 65M12, 65M06, 65M60.

Key words and phrases. Resolvent estimates, maximum-norm, elliptic, parabolic, finite elements.

The first author was partly supported by the Swiss National Science Foundation under Grant 20-56577.99.

The second and third authors were partly supported by the U.S. National Science Foundation under Grant DMS 0071412. 
(cf., in this respect, Da Prato and Sinestrari [9]). However, it was shown in [26] that if we restrict the considerations to $\mathcal{C}_{0}$, and thus also require $A v \in \mathcal{C}_{0}$ for $v$ to be in $D(A)$, then $A$ generates an analytic semigroup. This semigroup $E(t)=e^{-t A}$ may then be represented as

$$
E(t)=\frac{1}{2 \pi \mathrm{i}} \int_{\Gamma} e^{-\lambda t}(\lambda I-A)^{-1} d \lambda,
$$

where the contour $\Gamma$ may be chosen, e.g., as $\Gamma=\{\lambda ;|\arg \lambda|=\alpha\}$, where $\alpha \in$ $\left(\varphi, \frac{1}{2} \pi\right)$, with $\operatorname{Im} \lambda$ increasing from $-\infty$ to $\infty$. The semigroup $E(t)$ is the solution operator for the initial-value problem

$$
u_{t}=\Delta u \quad \text { in } \Omega, \quad u=0 \quad \text { on } \partial \Omega, \quad \text { with } u(0)=v, \quad \text { for } v \in \mathcal{C}_{0} .
$$

The maximum principle for the heat equation shows that $E(t)$ is a contraction semigroup in $\mathcal{C}_{0}$ with respect to $|\cdot|$, and the resolvent estimate (1.1) implies the smoothing property

$$
\left|E^{\prime}(t) v\right|=|\Delta E(t) v| \leq C t^{-1}|v|, \quad \text { for } t>0,
$$

by a classical theorem of Hille, cf. Pazy [17, Theorem 2.5.2. Conversely, by the same theorem, the smoothing property implies (1.1) for some $\varphi \in\left(0, \frac{1}{2} \pi\right)$.

We are interested in finite element analogues of these properties. From now on we assume that the basic domain $\Omega$ is convex. For $h \in\left(0, h_{0}\right], h_{0}>0$, we denote by $\mathcal{T}_{h}=\left\{\tau_{j}\right\}_{j=1}^{J_{h}}$ a triangulation of $\Omega_{h}=$ Int $\left(\bigcup_{j} \overline{\tau_{j}}\right) \subset \Omega$ into mutually disjoint open face-to-face simplices $\tau_{j}$. We assume that any vertex of a simplex $\tau_{j}$ which is on $\partial \Omega_{h}$ is also on $\partial \Omega$. Letting $h=\max _{j} \operatorname{diam} \tau_{j}$, we also assume that the family $\left\{\mathcal{T}_{h}\right\}$ of triangulations is globally quasi-uniform, so that $\min _{j} \operatorname{vol}\left(\tau_{j}\right) \geq c h^{d}$, with $c>0$. We note that then $\operatorname{dist}(x, \partial \Omega) \leq C h^{2}$ for $x \in \partial \Omega_{h}$.

Let $S_{h}$ be the finite dimensional space of continuous complex-valued piecewise linear functions associated with $\mathcal{T}_{h}$ that vanish outside $\Omega_{h}$. With the operator $A$ we associate its finite element version $A_{h}: S_{h} \rightarrow S_{h}$ by

$$
\left(A_{h} \psi, \chi\right)=(\nabla \psi, \nabla \chi), \quad \forall \psi, \chi \in S_{h}, \quad \text { where }(v, w)=\int_{\Omega} v \bar{w} d x .
$$

We remark that, since $A_{h} \psi \in S_{h}$, this function vanishes on $\partial \Omega$, which is analogous to the case when $v \in D(A)$ requires $A v \in \mathcal{C}_{0}$.

Consider now the spatially semidiscrete version of the initial-value problem (1.2),

$$
\left(u_{h, t}, \chi\right)+\left(\nabla u_{h}, \nabla \chi\right)=0, \quad \forall \chi \in S_{h}, t>0, \quad \text { with } u_{h}(0)=v_{h},
$$

or

$$
u_{h, t}+A_{h} u_{h}=0, \quad \text { for } t>0, \quad \text { with } u_{h}(0)=v_{h} .
$$

The solution operator $E_{h}(t)$ of this problem, defined by $u_{h}(t)=E_{h}(t) v_{h}$, is then the semigroup $E_{h}(t)=e^{-A_{h} t}$. A maximum-principle is not valid for the semidiscrete problem, and this raises the question about the boundedness of $E_{h}(t)$ in maximumnorm. In this regard it was shown in Thomée and Wahlbin 29] (following Schatz, Thomée, and Wahlbin [22]) that

$$
\left|E_{h}(t) v_{h}\right|+t\left|E_{h}^{\prime}(t) v_{h}\right| \leq C\left|v_{h}\right|, \quad \text { for } t>0 .
$$

By the theorem of Hille referred to above, this implies that the analogue of (1.1) is satisfied for $A_{h}$ and some $\varphi \in(0, \pi / 2)$. We note that the existence of a resolvent estimate for $A_{h}$ would provide an alternative proof of the stability and smoothing property (1.4). 
Our main result in this paper is now the following, with $\Omega$ a bounded, convex domain with smooth boundary.

Theorem 1.1. For any $\varphi \in\left(0, \frac{1}{2} \pi\right)$ there exists a constant $C$ such that

$$
\left|\left(\lambda I-A_{h}\right)^{-1} \chi\right| \leq C(1+|\lambda|)^{-1}|\chi|, \quad \text { for } \lambda \notin \Sigma_{\varphi}, \quad \chi \in S_{h} .
$$

For earlier work concerning maximum-norm stability, smoothing and resolvent estimates of finite element operators, we also refer to Fujii 11], Schatz, Thomée, and Wahlbin [21], Nitsche and Wheeler [15, Wahlbin [31], Thomée and Wahlbin [28], Rannacher [18], Chen [5], Crouzeix, Larsson, and Thomée [6], Palencia [16], Bakaev, Larsson, and Thomée [3], Bakaev [2], Crouzeix and Thomée [7; brief discussions of the above work are given in [22] and [2]. We note, in particular, that the result in the case $d=1$ follows from [6], and we may therefore restrict our considerations to $d \geq 2$ below.

We emphasize that our Theorem 1.1 is an improvement of the resolvent estimate that follows from (1.4) in that the angle $\varphi$ can be chosen arbitrarily small. This is of interest, e.g., in the study of such single step time stepping methods for (1.3) for which the fully discrete solution at time $t_{n}=n k$ can be thought of as $U^{n}=r\left(k A_{h}\right)^{n} v_{h}$, where $k$ denotes a constant time step. This approximation may then be represented in terms of the resolvent as

$$
r\left(k A_{h}\right)^{n}=r(\infty)^{n} I+\frac{1}{2 \pi \mathrm{i}} \int_{\Gamma}\left(r(k \lambda)^{n}-r(\infty)^{n}\right)\left(\lambda I-A_{h}\right)^{-1} d \lambda,
$$

where $\Gamma=\{\lambda ;|\arg \lambda|=\alpha\}$ with $\alpha \in(0, \theta)$, if $r(\lambda)$ is $A(\theta)$-stable. Our result thus makes the analysis of time stepping schemes possible for schemes based on $A(\theta)$-stable rational functions with small $\theta$, cf., e.g., Thomée [27], Chapter 8.

The analogue of Theorem 1.1] was shown in 2] in the case of Lagrange elements of polynomial degree at least two for $d=2$ and 3 . Using the techniques for large $|\lambda|$ in Section 3 below, the restriction to $d=2,3$ in 2 is easily removed. Moreover, the bound in the piecewise linear case in [2] contains a factor $(\log \log (1 / h))^{1 / 2+d / 4}$, and an essential contribution of the present paper is to remove this factor.

Our approach is inspired by Palencia [16], and uses resolvent estimates for the continuous problem together with certain properties of the $L_{2}$ and Ritz projections onto $S_{h}$. Such preliminary material is collected in Section 2 below, and the proof of Theorem 1.1 is then given in Section 3. An important ingredient in our proof is a maximum-norm stability property of the gradient of the Ritz projection. A proof of this is given in an Appendix. The restriction to $A=-\Delta$ is made for simplicity of presentation only, and the modifications needed for more general positive definite second order elliptic operators with smooth real-valued coefficients would not present any difficulties.

\section{Preliminaries}

In this section we collect some properties related to the finite element spaces $S_{h}$ and the above operator $A=-\Delta$ which will be used below in the proofs of our main results.

In what follows, we shall repeatedly apply the well-known inverse property

$$
|\chi|_{1, \tau} \leq C h^{-1}|\chi|_{\tau} \quad \text { for } h \in\left(0, h_{0}\right], \tau \in \mathcal{T}_{h}, \text { and } \chi \in S_{h},
$$

which follows by the quasi-uniformity of the triangulations $\mathcal{T}_{h}$. As a consequence, the same inequality holds for the full domain $\Omega_{h}$ as well. Here and below, when $s$ 
is an integer, $|\cdot|_{s, V}$ denotes the standard norm in $\mathcal{C}^{s}(V)$ or $W_{\infty}^{s}(V)$, the functions in $V$ with derivatives of order $s$ in $\mathcal{C}(\bar{V})$ or $L_{\infty}(V)$, respectively; $V$ will normally be omitted when $V=\Omega$. We will use the norm $|\cdot|_{s+\alpha}$ in $\mathcal{C}^{s+\alpha}$ when $s$ is an integer and $0<\alpha<1$. We shall also use the notation $\dot{\mathcal{C}}^{s}=\mathcal{C}^{s} \cap \mathcal{C}_{0}$ and $\dot{W}_{\infty}^{s}=W_{\infty}^{s} \cap \mathcal{C}_{0}$ for $s>0$.

Let $P_{h}$ denote the orthogonal $L_{2}$-projection onto $S_{h}$, so that

$$
\left(P_{h} v, \chi\right)=(v, \chi), \quad \forall \chi \in S_{h} .
$$

We shall need the stability of $P_{h}$ with respect to the maximum-norm (cf. Descloux [8], Douglas, Dupont and Wahlbin [10]),

$$
\left|P_{h} v\right| \leq C|v|_{\Omega_{h}}, \quad \text { for } v \in L_{\infty} .
$$

Using (2.1) and a well-known estimate for the standard Lagrange interpolant $I_{h}$, we easily find that

$$
\left|P_{h} v\right|_{1} \leq C|v|_{1, \Omega_{h}}, \quad \text { for } v \in \dot{W}_{\infty}^{1} .
$$

Next let $R_{h}$ be the standard Ritz projection onto $S_{h}$, given by

$$
\left(\nabla R_{h} v, \nabla \chi\right)=(\nabla v, \nabla \chi), \quad \forall \chi \in S_{h} .
$$

It is known from Schatz and Wahlbin [23] that, for small $h$,

$$
\left|R_{h} v\right| \leq C \log (1 / h)|v|_{\Omega_{h}}, \quad \text { for } v \in \mathcal{C},
$$

where the logarithm cannot be removed in our case of piecewise linear finite elements, see Haverkamp 12]. An essential component in our approach is the following stability estimate for $R_{h}$ in $W_{\infty}^{1}$, without a logarithmic factor:

$$
\left|R_{h} v\right|_{1} \leq C|v|_{1, \Omega_{h}}+C h|v|_{1, \Omega \backslash \Omega_{h}}, \quad \text { for } v \in \dot{W}_{\infty}^{1} .
$$

This was shown in Rannacher and Scott [19] in the case of a convex polygonal domain in the plane, and also stated there in the case of a curvilinear domain (cf. also Chen and Huang [4] for the case $d=3$ ). A proof in our case of arbitrary space dimension is given here in an Appendix.

Applying (2.5) to $I_{h} v-v$, we find, with $0<\alpha<1$, that

$$
\left|R_{h} v-v\right|_{1, \Omega_{h}} \leq C\left|I_{h} v-v\right|_{1, \Omega_{h}}+C h|v|_{1, \Omega \backslash \Omega_{h}} \leq C h^{\alpha}|v|_{1+\alpha}, \quad \text { for } v \in \dot{\mathcal{C}}^{1+\alpha},
$$

and, using the fact that $P_{h} v-R_{h} v=P_{h}\left(v-R_{h} v\right)$, it follows from (2.3) that

$$
\left|\left(P_{h}-R_{h}\right) v\right|_{1} \leq C\left|R_{h} v-v\right|_{1, \Omega_{h}} \leq C h^{\alpha}|v|_{1+\alpha}, \quad \text { for } v \in \dot{\mathcal{C}}^{1+\alpha}, \quad 0<\alpha<1 \text {. }
$$

We now turn to some resolvent estimates for the operator $A$ which will be needed below. We begin with the following result, which includes (1.1).

Theorem 2.1. For any $\varphi \in\left(0, \frac{1}{2} \pi\right)$, there exists a constant $C$ such that

$$
\left|(\lambda I-A)^{-1} v\right|_{j} \leq C(1+|\lambda|)^{-1+j / 2}|v|, \quad \text { for } \lambda \notin \Sigma_{\varphi}, \quad j=0,1, \quad v \in \mathcal{C} .
$$

Proof. It follows from Theorem 1 of [26] that the estimate of (2.7) holds for $\lambda \notin$ $\Sigma_{\varphi},|\lambda| \geq R$, for some $R \geq 0$ and some $\varphi \in\left(0, \frac{1}{2} \pi\right)$. To see that in the case $A=-\Delta$ the angle $\varphi$ may be chosen arbitrarily small, we observe that by the proof in [26] it suffices to note that for any homogeneous positive definite polynomial $p(\xi)$ in $R^{d}$ with real coefficients we have $\left|p(\xi)-e^{\mathrm{i} \theta} \tau^{2}\right| \geq c>0$ for $|\xi|^{2}+\tau^{2}=1,0<\varphi \leq|\theta| \leq \pi$. Here $p(\xi)$ is the characteristic polynomial of $A=-\Delta$ (i.e., $p(\xi)=|\xi|^{2}$ ) or that of the principal part of the operator after a transformation needed to make the boundary locally plane near a point $x \in \partial \Omega$. 
To complete the proof we need to show that $\left|(\lambda I-A)^{-1} v\right|_{1} \leq C|v|$ for $\lambda \notin$ $\Sigma_{\varphi},|\lambda| \leq R$. We first note that if

$$
\left\|(\lambda I-A)^{-1} v\right\|_{L_{p}} \leq\|v\|_{L_{p}}, \quad \text { for } \lambda \notin \Sigma_{\varphi},|\lambda| \leq R, \quad \text { with } p<\infty,
$$

then, using elliptic regularity (cf., e.g., Agmon, Douglis, and Nirenberg [1]) and the identity $A(\lambda I-A)^{-1}=-I+\lambda(\lambda I-A)^{-1}$,

$$
\left\|(\lambda I-A)^{-1} v\right\|_{W_{p}^{2}} \leq C\left\|A(\lambda I-A)^{-1} v\right\|_{L_{p}} \leq C\|v\|_{L_{p}} .
$$

Hence, by Sobolev's inequality, for $p^{-1}+q^{-1}<2 d^{-1}, q>p$,

$$
\left\|(\lambda I-A)^{-1} v\right\|_{L_{q}} \leq C\left\|(\lambda I-A)^{-1} v\right\|_{W_{p}^{2}} \leq C\|v\|_{L_{p}} \leq C\|v\|_{L_{q}} .
$$

For $p=2$ we have directly, with $\lambda_{0}$ the minimal eigenvalue of $A$, and $\lambda \notin \Sigma_{\varphi}$,

$$
\left\|(\lambda I-A)^{-1} v\right\|_{L_{2}} \leq C \sup _{x \geq \lambda_{0}} \frac{1}{|\lambda-x|}\|v\|_{L_{2}} \leq C\|v\|_{L_{2}} .
$$

Hence the above estimates follow for any $p<\infty$ in a finite number of steps. Finally, using the Sobolev inequality $|v|_{1} \leq C\|v\|_{W_{q}^{2}}$ with $q$ sufficiently large completes the proof of the desired result.

Theorem 2.2. For any $\varphi \in(0, \pi / 2)$, there exists a constant $C$ such that

$$
\left|A(\lambda I-A)^{-1} v\right| \leq C(1+|\lambda|)^{-1 / 2}|v|_{1}, \quad \text { for } \lambda \notin \Sigma_{\varphi}, v \in \dot{W}_{\infty}^{1} .
$$

Proof. Application of (2.7) with $j=0$ shows that

$$
\left|A(\lambda I-A)^{-1} v\right| \leq C|v|, \quad \text { for } v \in \mathcal{C}
$$

and, replacing $v$ by $A v$ in (2.7),

$$
\left|A(\lambda I-A)^{-1} v\right| \leq C(1+|\lambda|)^{-1}|A v| \leq C(1+|\lambda|)^{-1}|v|_{2}, \quad \text { for } v \in \dot{\mathcal{C}}^{2} .
$$

We now claim that, for $v \in \dot{W}_{\infty}^{1}$ and $t>0$ a parameter, we may write $v=$ $w_{0}(t)+w_{1}(t)$, with $w_{0}(t) \in \mathcal{C}_{0}, w_{1}(t) \in \dot{\mathcal{C}}^{2}$, such that

$$
\left|w_{0}(t)\right| \leq C t^{1 / 2}|v|_{1}, \quad\left|w_{1}(t)\right|_{2} \leq C t^{-1 / 2}|v|_{1}, \quad \text { for } t>0 .
$$

In fact, using a partition of unity and a change of variables, it suffices to show this in a halfplane, $x_{1}>0$, say, and for $v$ with compact support and with $v=0$ for $x_{1}=0$. To treat the function near the boundary, one may then extend $v$ by $v\left(-x_{1}, x_{2}, \ldots, x_{d}\right)=-v\left(x_{1}, x_{2}, \ldots, x_{d}\right)$ and then take for $w_{1}(t)$ a smoothing convolution of $v$ over a disc with radius $t^{1 / 2}$ with even kernel. This gives $w_{1}(t)=0$ for $x_{1}=0$, so that $w_{1}(t) \in \dot{\mathcal{C}}^{2}$ and (2.9) holds. With $t=(1+|\lambda|)^{-1}$ this shows that

$$
\left|A(\lambda I-A)^{-1} v\right| \leq C\left|w_{0}(t)\right|+C(1+|\lambda|)^{-1}\left|w_{1}(t)\right|_{2} \leq C(1+|\lambda|)^{-1 / 2}|v|_{1} .
$$

We shall also need a resolvent estimate in a Hölder norm. In the proof of this we shall use some notions from real interpolation theory, cf., e.g., Triebel [30]. When $A_{1} \subseteq A_{0}$ are two Banach spaces with $\|v\|_{A_{0}} \leq C\|v\|_{A_{1}}$ for $v \in A_{1}$, the space $\left(A_{0}, A_{1}\right)_{1 / 2, \infty}$ is defined by the norm

$$
\|v\|_{\left(A_{0}, A_{1}\right)_{1 / 2, \infty}}=\|v\|_{A_{0}}+\sup _{t>0} t^{-1 / 2} \inf _{\substack{v=w_{0}(t)+w_{1}(t) \\ w_{0}(t) \in A_{0}, w_{1}(t) \in A_{1}}}\left(\left\|w_{0}(t)\right\|_{A_{0}}+t\left\|w_{1}(t)\right\|_{A_{1}}\right) .
$$

If $L$ is a linear operator $A_{0} \rightarrow B_{0}$ and $A_{1} \rightarrow B_{1}$ with norms $N_{0}$ and $N_{1}$, respectively, then also $L:\left(A_{0}, A_{1}\right)_{1 / 2, \infty} \rightarrow\left(B_{0}, B_{1}\right)_{1 / 2, \infty}$, with norm bounded by $C N_{0}^{1 / 2} N_{1}^{1 / 2}$. From [30], Section 4.5, we have that $\left(\mathcal{C}^{\alpha}, \mathcal{C}^{2+\alpha}\right)_{1 / 2, \infty}=\mathcal{C}^{1+\alpha}$ for $0<\alpha<1$. 
Also, from (2.9) it follows that $\dot{W}_{\infty}^{1} \subseteq\left(\mathcal{C}, \dot{\mathcal{C}}^{2}\right)_{1 / 2, \infty}$ and $\|v\|_{\left(\mathcal{C}, \dot{\mathcal{C}}^{2}\right)_{1 / 2, \infty}} \leq C|v|_{1}$, for $v \in \dot{W}_{\infty}^{1}$

Theorem 2.3. For any $\varphi \in(0, \pi / 2)$ and $\alpha \in(0,1)$ there exists a constant $C$ such that

$$
\left|(\lambda I-A)^{-1} v\right|_{1+\alpha} \leq C(1+|\lambda|)^{-1+\alpha / 2}|v|_{1}, \quad \text { for } \lambda \notin \Sigma_{\varphi}, v \in \dot{W}_{\infty}^{1} .
$$

Proof. We start with the estimate

$$
\left|(\lambda I-A)^{-1} v\right|_{\alpha} \leq C(1+|\lambda|)^{-1+\alpha / 2}|v|, \quad \text { for } \lambda \notin \Sigma_{\varphi}, v \in \mathcal{C} .
$$

In fact, this holds for $\alpha=0$ and $\alpha=1$ by (2.7), and hence follows for $0<\alpha<1$ since $|w|_{\alpha} \leq C|w|_{1}^{\alpha}|w|^{1-\alpha}$. Applying (2.11) with $A v$ substituted for $v$, we find that

$$
\left|(\lambda I-A)^{-1} A v\right|_{\alpha} \leq C(1+|\lambda|)^{-1+\alpha / 2}|A v|, \quad \text { for } \lambda \notin \Sigma_{\varphi}, v \in \dot{\mathcal{C}}^{2} .
$$

By a Schauder estimate for the elliptic operator $A$, cf. [1, we have, for $0<\alpha<1$,

$$
\left|(\lambda I-A)^{-1} v\right|_{2+\alpha} \leq C\left|A(\lambda I-A)^{-1} v\right|_{\alpha}=C\left|(\lambda I-A)^{-1} A v\right|_{\alpha}, \quad \text { for } v \in \dot{\mathcal{C}}^{2},
$$

so that by (2.12),

$$
\left|(\lambda I-A)^{-1} v\right|_{2+\alpha} \leq C(1+|\lambda|)^{-1+\alpha / 2}|v|_{2}, \quad \text { for } \lambda \notin \Sigma_{\varphi}, v \in \dot{\mathcal{C}}^{2} .
$$

Thus by (2.11) and (2.13) $(\lambda I-A)^{-1}$ is a bounded operator both $\mathcal{C} \rightarrow \mathcal{C}^{\alpha}$ and $\dot{\mathcal{C}}^{2} \rightarrow \mathcal{C}^{2+\alpha}$, with norms bounded by the same number in the way shown. From our remarks above about real interpolation theory, 2.10) hence follows by taking $A_{0}=\mathcal{C}, A_{1}=\dot{\mathcal{C}}^{2}, B_{0}=\mathcal{C}^{\alpha}$, and $B_{1}=\mathcal{C}^{2+\alpha}$.

\section{Proof of the main Result}

In this section we give the proof of Theorem 1.1 as stated in the introduction. The proof will be separated into two parts, involving different techniques. The first part concerns $|\lambda| \leq \omega_{0} h^{-2}$, with $\omega_{0}$ sufficiently small, and the second part treats $|\lambda|>\omega_{0} h^{-2}$.

For the first part we shall show that there exists $\omega_{0}>0$ such that, for any $\varphi \in\left(0, \frac{1}{2} \pi\right)$ and $\chi \in S_{h}$,

$$
\left|A_{h}\left(\lambda I-A_{h}\right)^{-1} \chi\right| \leq C(1+|\lambda|)^{-1 / 2}|\chi|_{1}, \quad \text { for } \lambda \notin \Sigma_{\varphi},|\lambda| \leq \omega_{0} h^{-2} .
$$

We now show that (3.1) implies the result of Theorem 1.1 for these $\lambda$. Since $P_{h} A=A_{h} R_{h}$, we have, for $\chi \in S_{h}$, the identity

$$
\left(\lambda I-A_{h}\right)^{-1} \chi=P_{h}(\lambda I-A)^{-1} \chi+A_{h}\left(\lambda I-A_{h}\right)^{-1}\left(P_{h}-R_{h}\right)(\lambda I-A)^{-1} \chi,
$$

where both sides are well defined for $\lambda \notin \Sigma_{\varphi}$. Using the estimates (2.2), (2.7) with $j=0$, and (3.1), we find that

$$
\begin{aligned}
& \left|\left(\lambda I-A_{h}\right)^{-1} \chi\right| \leq C(1+|\lambda|)^{-1}|\chi| \\
& +C(1+|\lambda|)^{-1 / 2}\left|\left(P_{h}-R_{h}\right)(\lambda I-A)^{-1} \chi\right|_{1}, \quad \text { for } \lambda \notin \Sigma_{\varphi}, \quad \chi \in S_{h} .
\end{aligned}
$$

In view of (2.3), (2.5), and (2.7) with $j=1$, this shows the desired result.

A similar approach was used in [16]; it is the fact that we use $|\cdot|_{1}$ in the last term in (3.2) that makes it possible to avoid the logarithm from (2.4).

To show (3.1) we now use the identity

$$
\begin{aligned}
& A_{h}\left(\lambda I-A_{h}\right)^{-1} \chi=P_{h} A(\lambda I-A)^{-1} \chi \\
& \quad+\lambda A_{h}\left(\lambda I-A_{h}\right)^{-1}\left(P_{h}-R_{h}\right)(\lambda I-A)^{-1} \chi, \quad \text { for } \lambda \notin \Sigma_{\varphi}, \chi \in S_{h} .
\end{aligned}
$$


Here, by (2.2) and (2.8),

$$
\left|P_{h} A(\lambda I-A)^{-1} \chi\right| \leq C(1+|\lambda|)^{-1 / 2}|\chi|_{1}, \quad \text { for } \lambda \notin \Sigma_{\varphi} .
$$

Using the operator norm $\left|B_{h}\right|_{S_{h}, 1}=\sup _{\chi \in S_{h}}\left(\left|B_{h} \chi\right| /|\chi|_{1}\right)$, we see that the last term in (3.3) is bounded by

$$
C|\lambda|\left|A_{h}\left(\lambda I-A_{h}\right)^{-1}\right|_{S_{h}, 1}\left|\left(P_{h}-R_{h}\right)(\lambda I-A)^{-1} \chi\right|_{1} .
$$

To bound the last factor we have, by (2.6) and (2.10),

$$
\left|\left(P_{h}-R_{h}\right)(\lambda I-A)^{-1} \chi\right|_{1} \leq C h^{1 / 2}\left|(\lambda I-A)^{-1} \chi\right|_{3 / 2} \leq C h^{1 / 2}(1+|\lambda|)^{-3 / 4}|\chi|_{1},
$$

so that (3.3) implies, for $\lambda \notin \Sigma_{\varphi}$,

$$
\left|A_{h}\left(\lambda I-A_{h}\right)^{-1}\right|_{S_{h}, 1} \leq C(1+|\lambda|)^{-1 / 2}+C_{1} h^{1 / 2}(1+|\lambda|)^{1 / 4}\left|A_{h}\left(\lambda I-A_{h}\right)^{-1}\right|_{S_{h}, 1} .
$$

Hence, if $C_{1} h^{1 / 2}(1+|\lambda|)^{1 / 4} \leq 1 / 2$, we find that

$$
\left|A_{h}\left(\lambda I-A_{h}\right)^{-1}\right|_{S_{h}, 1} \leq C(1+|\lambda|)^{-1 / 2}, \quad \lambda \notin \Sigma_{\varphi},
$$

which thus shows (3.1) for $|\lambda| \leq \omega_{0} h^{-2}$, for $h$ small and some $\omega_{0}>0$.

We now turn to the case $|\lambda| \geq \omega_{0} h^{-2}$, where $\omega_{0}$ is determined by the above. Let us note in passing that by the well-known inverse estimate $\left|A_{h} \chi\right| \leq C h^{-2}|\chi|$ for $\chi \in S_{h}$ we have $\left|\lambda^{-1} A_{h}\right|_{S_{h}} \leq 1 / 2$ for $|\lambda| \geq \omega_{1} h^{-2}$ with $\omega_{1}$ large enough, where $\left|A_{h}\right|_{S_{h}}=\sup _{\chi \in S_{h}}\left|A_{h} \chi\right| /|\chi|$. Hence $\left|\left(I-\lambda^{-1} A_{h}\right)^{-1}\right|_{S_{h}} \leq 2$ for $|\lambda| \geq \omega_{1} h^{-2}$, which implies (1.5) for $|\lambda| \geq \omega_{1} h^{-2}$, but leaves the possible gap $\omega_{0} h^{-2} \leq|\lambda| \leq \omega_{1} h^{-2}$.

For $x \in \Omega_{h}$ fixed and $\varphi \in\left(0, \frac{1}{2} \pi\right)$, we will use the adjoint discrete Green's function

$$
G_{h}^{x}(y, \bar{\lambda})=\left(\left(\bar{\lambda} I-A_{h}\right)^{-1} \delta_{h}^{x}\right)(y), \quad \text { for } \lambda \notin \Sigma_{\varphi},
$$

where $\delta_{h}^{x}$ is the discrete delta-function defined by

$$
\left(\chi, \delta_{h}^{x}\right)=\chi(x), \quad \forall \chi \in S_{h} .
$$

We then have

$$
\left(\left(\lambda I-A_{h}\right)^{-1} \chi\right)(x)=\left(\chi, G_{h}^{x}(\cdot, \bar{\lambda})\right), \quad \forall \chi \in S_{h} .
$$

Since the sector $\Sigma_{\varphi}$ is symmetric around the real axis, it therefore suffices to show that for any $x \in \Omega_{h}$ and $\varphi \in(0, \pi / 2)$, we have

$$
\left\|G_{h}^{x}(\cdot, \lambda)\right\|_{L_{1}} \leq C|\lambda|^{-1}, \quad \text { for }|\lambda| \geq \omega_{0} h^{-2}, \lambda \notin \Sigma_{\varphi} .
$$

Writing for brevity $\|\cdot\|=\|\cdot\|_{L_{2}(\Omega)}$, we introduce the weighted norm

$$
\|v\|_{m}=\left\|\left(\rho_{h}^{x}\right)^{m} v\right\|, \quad m \geq 0, \quad \text { where } \rho_{h}^{x}(y)=\left(|x-y|^{2}+h^{2}\right)^{1 / 2} .
$$

The estimate (3.4) will then be a consequence of the following lemma.

Lemma 3.1. For each $m \geq 0, \varphi \in(0, \pi / 2)$, and $\omega_{0}>0$ there is a $C$ such that for any $x \in \Omega_{h}$ we have

$$
\left\|G_{h}^{x}(\cdot, \lambda)\right\|_{m} \leq C h^{m-d / 2}|\lambda|^{-1}, \quad \text { for } \lambda \notin \Sigma_{\varphi}, \quad|\lambda| \geq \omega_{0} h^{-2} .
$$

In fact, choosing $m>d / 2$, we have $\left\|\left(\rho_{h}^{x}\right)^{-m}\right\| \leq C h^{-m+d / 2}$, and hence

$$
\left\|G_{h}^{x}(\cdot, \lambda)\right\|_{L_{1}} \leq C\left\|\left(\rho_{h}^{x}\right)^{-m}\right\|\left\|G_{h}^{x}(\cdot, \lambda)\right\|_{m} \leq\left(C h^{-m+d / 2}\right)\left(C h^{m-d / 2}|\lambda|^{-1}\right)=C|\lambda|^{-1} .
$$

For the proof of Lemma 3.1 we shall need the following two lemmas.

Lemma 3.2. For each $m \geq 0$ there is a $C$ such that for any $x \in \Omega_{h}$ we have

$$
\left\|\delta_{h}^{x}\right\|_{m} \leq C h^{m-d / 2} \text {. }
$$


Proof. Using the exponential decay property of $\delta_{h}^{x}$, see [8], [10], this follows easily as in [21], Lemma 1.5.

Lemma 3.3. For each $m \geq 1$ there is a $C$ such that for any $x \in \Omega_{h}$ and $\chi \in S_{h}$ we have

$$
\left\|\rho^{-m+1} \nabla\left(\rho^{2 m} \chi-P_{h}\left(\rho^{2 m} \chi\right)\right)\right\| \leq C h\left(\|\chi\|_{m-1}+\|\nabla \chi\|_{m}\right), \quad \text { where } \rho=\rho_{h}^{x} .
$$

This is a special case of [2], Lemma 3.4 (with $j=1$ ).

Proof of Lemma 3.1. For brevity we write $G$ for $G_{h}^{x}$. We consider the expression (3.6)

$$
-\lambda\|G\|_{m}^{2}+\|\nabla G\|_{m}^{2}=-\lambda\left(G, \rho^{2 m} G\right)+\left(\nabla G, \nabla\left(\rho^{2 m} G\right)\right)-2 m\left(\nabla G, \rho^{2 m-1} \nabla \rho G\right) .
$$

We note that $G$ satisfies

$$
-\lambda(G, \chi)+(\nabla G, \nabla \chi)=-\left(\delta_{h}^{x}, \chi\right), \quad \forall \chi \in S_{h} .
$$

Choosing $\chi=P_{h}\left(\rho^{2 m} G\right)$ and subtracting from (3.6)

$$
\begin{aligned}
-\lambda\|G\|_{m}^{2}+\|\nabla G\|_{m}^{2}=F:= & \left(\nabla G, \nabla\left(\rho^{2 m} G-P_{h}\left(\rho^{2 m} G\right)\right)\right) \\
& +\left(\delta_{h}^{x}, \rho^{2 m} G\right)-2 m\left(\nabla G, \rho^{2 m-1} \nabla \rho G\right) .
\end{aligned}
$$

This equation is of the form

$$
e^{\mathrm{i} \alpha} a+b=f, \quad \text { with } a, b>0,0 \leq|\alpha| \leq \pi-\varphi,
$$

and on multiplying by $e^{-\mathrm{i} \alpha / 2}$ and taking real parts we have

$$
a+b \leq(\cos (\alpha / 2))^{-1}|f| \leq(\sin (\varphi / 2))^{-1}|f|=C_{\varphi}|f| .
$$

From (3.7) we therefore conclude that

$$
|\lambda|\|G\|_{m}^{2} \leq|\lambda|\|G\|_{m}^{2}+\|\nabla G\|_{m}^{2} \leq C_{\varphi}|F|, \quad \text { for } \lambda \notin \Sigma_{\varphi} .
$$

For $m=0$ we note that $F=\left(\delta_{h}^{x}, G\right)$ and hence, by (3.8) and Lemma 3.2,

$$
|\lambda|\|G\|_{0} \leq C\left\|\delta_{h}^{x}\right\| \leq C h^{-d / 2} .
$$

Now let $m \geq 1$. By Lemma 3.3 and using the easily shown inverse inequality $h\|\nabla \chi\|_{m} \leq C\|\chi\|_{m}$, and the fact that $h \leq \rho$, we then have

$$
\left\|\rho^{-m+1} \nabla\left(\rho^{2 m} G-P_{h}\left(\rho^{2 m} G\right)\right)\right\| \leq C h\left(\|G\|_{m-1}+\|\nabla G\|_{m}\right) \leq C\|G\|_{m},
$$

and hence, for the first term in $F$,

$$
\left|\left(\nabla G, \nabla\left(\rho^{2 m} G-P_{h}\left(\rho^{2 m} G\right)\right)\right)\right| \leq C\|\nabla G\|_{m-1}\|G\|_{m} .
$$

Further, by Lemma 3.2,

$$
\left|\left(\delta_{h}^{x}, \rho^{2 m} G\right)\right| \leq\left\|\delta_{h}^{x}\right\|_{m}\|G\|_{m} \leq C h^{m-d / 2}\|G\|_{m},
$$

and, since $\nabla \rho$ is bounded,

$$
\left|\left(\nabla G, \rho^{2 m-1} \nabla \rho G\right)\right| \leq C\|\nabla G\|_{m-1}\|G\|_{m} .
$$

Thus, from (3.8),

$$
|\lambda|\|G\|_{m} \leq C\left(\|\nabla G\|_{m-1}+h^{m-d / 2}\right) .
$$

Using Hölder's and Young's inequalities, we next find that

$$
\begin{aligned}
C\|\nabla G\|_{m-1} & \leq C h^{-1}\|G\|_{m-1} \leq C h^{-1}\|G\|_{m}^{1-1 / m}\|G\|_{0}^{1 / m} \\
& \leq \frac{1}{2}|\lambda|\|G\|_{m}+C|\lambda|^{-m+1} h^{-m}\|G\|_{0} \leq \frac{1}{2}|\lambda|\|G\|_{m}+C h^{m-2}\|G\|_{0},
\end{aligned}
$$


where we have used $|\lambda| \geq \omega_{0} h^{-2}$ in the last step. Hence, from (3.9) and (3.10),

$$
|\lambda|\|G\|_{m} \leq C\left(h^{m-2}\|G\|_{0}+h^{m-d / 2}\right) \leq C\left(h^{m-2-d / 2}|\lambda|^{-1}+h^{m-d / 2}\right) \leq C h^{m-d / 2} .
$$

This completes the proof for $m \geq 1$, and for $0<m<1$ the result is then obvious.

We remark that (3.1) is a discrete analogue of Theorem 2.2, which could possibly be of independent interest; the inequality holds also for $|\lambda| \geq \omega_{0} h^{-2}$, which easily follows from Theorem 1.1 since, by the inverse estimate $\left|A_{h} \chi\right| \leq C h^{-1}|\chi|_{1}$ on $S_{h}$, we may conclude

$$
\begin{aligned}
\left|A_{h}\left(\lambda I-A_{h}\right)^{-1} \chi\right| & =\left|\left(\lambda I-A_{h}\right)^{-1} A_{h} \chi\right| \leq 2|\lambda|^{-1}\left|A_{h} \chi\right| \\
& \leq C h^{-1}|\lambda|^{-1}|\chi|_{1} \leq C|\lambda|^{-1 / 2}|\chi|_{1}, \quad \text { for }|\lambda| \geq \omega_{0} h^{-2} .
\end{aligned}
$$

\section{ApPendix. MAXimum-NORM STABILITY OF THE GRADIENT OF THE RITZ PROJECTION}

We now show a stability estimate for $\nabla R_{h}$ which implies the estimate (2.5) needed in our proof of the resolvent estimate above. For the purpose of possible future reference we include, following Schatz [20, a weight function which shows a certain local character of the result, which is not needed here. As before, we use $\rho_{h}^{x}(y)=\left(|y-x|^{2}+h^{2}\right)^{1 / 2}$.

Theorem A.1. Let $0 \leq s<1$. There exists a constant $C$ such that for all $x \in \Omega_{h}$,

$$
\left|\nabla R_{h} v(x)\right| \leq C\left(\left|\left(h / \rho_{h}^{x}\right)^{s} \nabla v\right|_{\Omega_{h}}+\left\|\left(\rho_{h}^{x}\right)^{-d} v\right\|_{L_{1}\left(\partial \Omega_{h}\right)}\right), \quad \text { for } v \in W_{\infty}^{1} .
$$

To show (2.5) we note that, using polar coordinates,

$$
\left\|\left(\rho_{h}^{x}\right)^{-d}\right\|_{L_{1}\left(\partial \Omega_{h}\right)} \leq C \int_{0}^{\infty} r^{d-2}\left(r^{2}+h^{2}\right)^{-d / 2} d r+C \leq C h^{-1} .
$$

Since also $|v|_{\partial \Omega_{h}} \leq C h^{2}|\nabla v|_{\Omega \backslash \Omega_{h}}$ for $v \in \dot{W}_{\infty}^{1}$, the last term in (A.1) is bounded as desired. Using (A.1) with $s=0$ therefore shows (2.5).

Proof of Theorem A.1. Let $x$ be an arbitrary point in $\Omega_{h}$; this point will be fixed below. Denote by $\partial$ any derivative of first order, and let $\tau \in \mathcal{T}_{h}$ be such that $x \in \tau$. Let $\tilde{\delta}$ be a smooth nonnegative function with support in $\sigma \subset \tau$, with $\int_{\sigma} \tilde{\delta} d y=1$ and such that

$$
\operatorname{dist}\left(\sigma, \partial \Omega_{h}\right) \geq c h, \quad \text { with } c>0 .
$$

Furthermore, $\tilde{\delta}$ can be taken so that $\sigma$ contains a ball of radius $\geq c h$, and

$$
|\tilde{\delta}(y)| \leq C h^{-d} .
$$

Writing $R_{h} v=v_{h}$, we have, since $\partial v_{h}$ is constant on $\tau$,

$$
\partial v_{h}(x)=\left(\partial v_{h}, \tilde{\delta}\right)=-\left(v_{h}, \partial \tilde{\delta}\right) .
$$

Let $g=g^{x}(y)$ be the solution of

$$
-\Delta g=\partial \tilde{\delta} \quad \text { in } \Omega, \quad \text { with } g=0 \quad \text { on } \partial \Omega,
$$

and let $g_{h}=R_{h} g$ be its Ritz projection which satisifies $-\Delta_{h} g_{h}=P_{h}(\partial \tilde{\delta})$. Then, continuing from (A.5), with $(v, w)_{V}=\int_{V} v \bar{w} d x$,

$$
\partial v_{h}(x)=\left(\nabla v_{h}, \nabla g_{h}\right)=\left(\nabla v, \nabla g_{h}\right)=\left(\nabla v, \nabla\left(g_{h}-g\right)\right)_{\Omega_{h}}+(\nabla v, \nabla g)_{\Omega_{h}} .
$$


Here, by Green's formula and (A.6),

$$
(\nabla v, \nabla g)_{\Omega_{h}}=-(v, \partial \tilde{\delta})_{\Omega_{h}}+\left(v, \frac{\partial g}{\partial n}\right)_{\partial \Omega_{h}}=(\partial v, \tilde{\delta})_{\sigma}+\left(v, \frac{\partial g}{\partial n}\right)_{\partial \Omega_{h}} .
$$

With $G=G^{y}(\cdot)$ now denoting the standard Green's function for Poisson's equation with homogeneous Dirichlet boundary conditions, we have

$$
g(y)=\left(G^{y}, \partial \tilde{\delta}\right)_{\sigma}=-\left(\partial G^{y}, \tilde{\delta}\right)_{\sigma} .
$$

We shall use the following result from Krasovskii [13].

Lemma A.1. For $|\alpha|+|\beta|>0$ there exists $C$ such that

$$
\left|D_{y}^{\alpha} D_{z}^{\beta} G^{y}(z)\right| \leq C|z-y|^{-d+2-|\alpha|-|\beta|} .
$$

Combined with (A.9), we then find that, for $\operatorname{dist}(y, \sigma) \geq c h$,

$$
\left|D^{\alpha} g(y)\right| \leq C|y-x|^{-d+1-|\alpha|} .
$$

By (A.3) it follows that (for brevity we write $\rho=\rho_{h}^{x}$ below) $|\nabla g(y)| \leq C \rho(y)^{-d}$ for $y \in \partial \Omega_{h}$. Using this in (A.8), together with a trivial estimate for the first term on the right there, and then inserting the result into (A.7), we now have

$$
\left|\partial v_{h}(x)\right| \leq\left|\left(\nabla v, \nabla\left(g_{h}-g\right)\right)_{\Omega_{h}}\right|+|\partial v|_{\sigma}+C\left\|\rho^{-d} v\right\|_{L_{1}\left(\partial \Omega_{h}\right)} .
$$

Since clearly $|\partial v|_{\sigma} \leq C\left|(h / \rho)^{s} \nabla v\right|_{\Omega_{h}}$, in order to conclude the proof of the theorem it remains to show that $\left|\left(\nabla v, \nabla\left(g_{h}-g\right)\right)_{\Omega_{h}}\right| \leq C\left|(h / \rho)^{s} \nabla v\right|_{\Omega_{h}}$, or

$$
\left\|\omega^{s} \nabla\left(g_{h}-g\right)\right\|_{L_{1}\left(\Omega_{h}\right)} \leq C, \quad \text { where } \omega(y)=\omega_{h}(y)=\rho(y) / h .
$$

For this we shall use the techniques developed in Scott [24], and in [23] and [20]. With $x$ the central point, we introduce the dyadic annuli

$$
\Omega_{j}=\Omega_{h} \cap A_{j}, \quad \text { where } A_{j}=\left\{y \in R^{d}: d_{j} \leq|y-x| \leq d_{j-1}\right\}, \quad \text { with } d_{j}=2^{-j} .
$$

This dyadic decomposition is broken off at a suitable distance from $x$; we let the innermost domain be $\Omega^{I}=\left\{y \in \Omega_{h}:|y-x| \leq M h\right\}$, where $M$ in the end is going to be chosen large enough. We thus have $\Omega_{h}=\Omega^{I} \cup\left(\bigcup_{j=j_{0}}^{\bar{j}_{h}} \Omega_{j}\right)$ with $j_{0}=\lceil-\log \operatorname{diam}(\Omega) / \log 2\rceil$ fixed and $\bar{j}_{h}=\lceil-\log (M h) / \log 2\rceil$, where $\lceil a\rceil$ denotes the smallest integer $\geq a$. We also use the notation $A_{j}^{\prime}=A_{j-1} \cup A_{j} \cup A_{j+1}, A_{j}^{\prime \prime}=$ $\left(A_{j}^{\prime}\right)^{\prime}, A_{j}^{\prime \prime \prime}=\left(A_{j}^{\prime \prime}\right)^{\prime}$, and analogously for $\Omega_{j}^{\prime}, \Omega_{j}^{\prime \prime}$, and $\Omega_{j}^{\prime \prime \prime}$.

In order to show A.11) we now consider the contributions of the various subregions $\Omega^{I}$ and $\Omega_{j}$. For $y \in \Omega^{I}$ we have $\omega(y)=\rho(y) / h \leq 1+M$. Thus, using also the Cauchy-Schwarz inequality (writing again $\|\cdot\|=\|\cdot\|_{L_{2}}$ ),

$$
\left\|\omega^{s} \nabla\left(g_{h}-g\right)\right\|_{L_{1}\left(\Omega^{I}\right)} \leq C(1+M)^{s}(M h)^{d / 2}\left(\left\|\nabla g_{h}\right\|+\|\nabla g\|\right) .
$$

An easy energy argument using (A.4 establishes that

$$
\left\|\nabla g_{h}\right\|^{2} \leq\|\nabla g\|^{2}=(g, \partial \tilde{\delta})=-(\partial g, \tilde{\delta}) \leq\|\nabla g\|\|\tilde{\delta}\| \leq C h^{-d / 2}\|\nabla g\|,
$$

and hence

$$
\left\|\omega^{s} \nabla\left(g_{h}-g\right)\right\|_{L_{1}\left(\Omega^{I}\right)} \leq K:=C(1+M)^{s} M^{d / 2} .
$$

We now turn to the contributions of the $\Omega_{j}$. With $\omega_{j}=d_{j} / h$ we have, by the Cauchy-Schwarz inequality (all summations in $j$ below are understood to be between $j_{0}$ and $\bar{j}_{h}$ ),

$$
\sum_{j}\left\|\omega^{s} \nabla\left(g_{h}-g\right)\right\|_{L_{1}\left(\Omega_{j}\right)} \leq C \sum_{j} \omega_{j}^{s} d_{j}^{d / 2}\left\|\nabla\left(g_{h}-g\right)\right\|_{\Omega_{j}}, \quad \text { with }\|\cdot\|_{V}=\|\cdot\|_{L_{2}(V)} .
$$


We shall use the following result, which shows that, locally, the energy error in the Ritz projection is bounded by local best approximation and the error itself measured in a weaker norm. The result is essentially due to Nitsche and Schatz [14], and is proved in 23] in our case when domains may impinge on the mesh boundary $\partial \Omega_{h}$ (cf. in particular [23], Theorem 4.1). For a domain $V \subset \Omega_{h}$, we let $V_{\gamma}=\left\{y \in \Omega_{h}: \operatorname{dist}(y, V) \leq \gamma\right\}$.

Lemma A.2. There exist $C$ and $c>0$ such that for $\gamma \geq$ ch and any $\chi \in S_{h}$,

$$
\left\|\nabla\left(R_{h} v-v\right)\right\|_{V} \leq C\left(\|\nabla(v-\chi)\|_{V_{\gamma}}+\gamma^{-1}\|v-\chi\|_{V_{\gamma}}+\gamma^{-1}\left\|R_{h} v-v\right\|_{V_{\gamma}}\right) .
$$

We shall see that Lemma A.2 and standard approximation theory imply

$$
\left\|\nabla\left(g_{h}-g\right)\right\|_{\Omega_{j}} \leq C h d_{j}^{-d / 2-1}+C d_{j}^{-1}\left\|g_{h}-g\right\|_{\Omega_{j}^{\prime}} .
$$

After this we shall use a duality argument to show that

$$
\left\|g_{h}-g\right\|_{\Omega_{j}^{\prime}} \leq C h d_{j}^{-d / 2}\left(\left\|\nabla\left(g_{h}-g\right)\right\|_{L_{1}\left(\Omega_{h}\right)}+1\right)+C h\left\|\nabla\left(g_{h}-g\right)\right\|_{\Omega_{j}^{\prime \prime \prime}} .
$$

Together, these inequalities show that

$$
\begin{aligned}
\sum_{j} \omega_{j}^{s} d_{j}^{d / 2}\left\|\nabla\left(g_{h}-g\right)\right\|_{\Omega_{j}} \leq & C h \sum_{j} \omega_{j}^{s} d_{j}^{-1}\left(\left\|\nabla\left(g_{h}-g\right)\right\|_{L_{1}\left(\Omega_{h}\right)}+1\right) \\
& +C h \sum_{j} \omega_{j}^{s} d_{j}^{d / 2-1}\left\|\nabla\left(g_{h}-g\right)\right\|_{\Omega_{j}^{\prime \prime \prime} .}
\end{aligned}
$$

Since $d_{\bar{j}_{h}} \geq M h$ and $s<1$, we have

$$
h \sum_{j} \omega_{j}^{s} d_{j}^{-1}=\sum_{j}\left(d_{j} / h\right)^{s-1} \leq C M^{s-1},
$$

and we conclude that

$$
\begin{aligned}
\sum_{j} \omega_{j}^{s} d_{j}^{d / 2}\left\|\nabla\left(g_{h}-g\right)\right\|_{\Omega_{j}} \leq & C M^{s-1}\left(\left\|\nabla\left(g_{h}-g\right)\right\|_{L_{1}\left(\Omega_{h}\right)}+1\right) \\
& +C M^{-1} \sum_{j} \omega_{j}^{s} d_{j}^{d / 2}\left\|\nabla\left(g_{h}-g\right)\right\|_{\Omega_{j}^{\prime \prime \prime}}
\end{aligned}
$$

In the last term we may replace $\Omega_{j}^{\prime \prime \prime}$ by $\Omega_{j}$ by increasing $C$ and observing that for the $\Omega_{j}$ that intersect $\Omega^{I},\left\|\nabla\left(g_{h}-g\right)\right\|_{\Omega_{j}} \leq C h^{-d / 2}$ by (A.12), so that their contribution may be added to the first term on the right. Thus

$$
\begin{aligned}
& \sum_{j} \omega_{j}^{s} d_{j}^{d / 2}\left\|\nabla\left(g_{h}-g\right)\right\|_{\Omega_{j}} \\
& \left.\leq C M^{s-1}\left(\left\|\nabla\left(g_{h}-g\right)\right\|_{L_{1}\left(\Omega_{h}\right)}+M^{d / 2}\right)+C M^{-1} \sum_{j} \omega^{s} d_{j}^{d / 2}\left\|\nabla\left(g_{h}-g\right)\right\|_{\Omega_{j}}\right),
\end{aligned}
$$

and choosing $M$ large enough we can kick back the last term to obtain

$$
\sum_{j} \omega_{j}^{s} d_{j}^{d / 2}\left\|\nabla\left(g_{h}-g\right)\right\|_{\Omega_{j}} \leq C M^{s-1}\left(\left\|\nabla\left(g_{h}-g\right)\right\|_{L_{1}\left(\Omega_{h}\right)}+M^{d / 2}\right) .
$$

It follows that

$$
\begin{aligned}
\left\|\omega^{s} \nabla\left(g_{h}-g\right)\right\|_{L_{1}\left(\Omega_{h}\right)} & \leq K+C \sum_{j} \omega_{j}^{s} d_{j}^{d / 2}\left\|\nabla\left(g_{h}-g\right)\right\|_{\Omega_{j}} \\
& \leq K+C M^{s-1}\left(\left\|\nabla\left(g_{h}-g\right)\right\|_{L_{1}\left(\Omega_{h}\right)}+M^{d / 2}\right) .
\end{aligned}
$$


Since $\left\|\nabla\left(g_{h}-g\right)\right\|_{L_{1}\left(\Omega_{h}\right)} \leq\left\|\omega^{s} \nabla\left(g_{h}-g\right)\right\|_{L_{1}\left(\Omega_{h}\right)}$, we may now kick back also the weighted $L_{1}\left(\Omega_{h}\right)$-norm, which would complete the proof of A.11) and hence of Theorem A.1.

It remains to prove (A.14) and A.15), and we begin with A.14). In Lemma A.2 we take $V=\Omega_{j}, \gamma=d_{j}, V_{\gamma}=\Omega_{j}^{\prime}$, and $\chi=I_{h} g$, the standard interpolant into $S_{h}$. We have, using (A.10),

$$
\left\|\nabla\left(g-I_{h} g\right)\right\|_{\Omega_{j}^{\prime}} \leq C d_{j}^{d / 2}\left|\nabla\left(g-I_{h} g\right)\right|_{\Omega_{j}^{\prime}} \leq C d_{j}^{d / 2} h\|g\|_{W_{\infty}^{2}\left(\Omega_{j}^{\prime \prime}\right)} \leq C d_{j}^{-d / 2-1} h .
$$

The same estimate is also valid for $d_{j}^{-1}\left\|g-I_{h} g\right\|_{L_{2}\left(\Omega_{j}^{\prime}\right)}$, which shows (A.14).

For (A.15) we consider $\left(g_{h}-g, \varphi\right)$ with $\|\varphi\|=1$, supp $\varphi \subset \Omega_{j}^{\prime}$. For each such $\varphi$, let $w$ be the solution of

$$
-\Delta w=\varphi \quad \text { in } \Omega, \quad \text { with } w=0 \quad \text { on } \partial \Omega .
$$

Then, for any $\chi \in S_{h}$, with $\Gamma_{h}=\Omega \backslash \Omega_{h}$ the "skin" layer,

$$
\begin{aligned}
\left(g_{h}-g, \varphi\right) & =\left(\nabla\left(g_{h}-g\right), \nabla w\right)=\left(\nabla\left(g_{h}-g\right), \nabla(w-\chi)\right) \\
& =\left(\nabla\left(g_{h}-g\right), \nabla(w-\chi)\right)_{\Omega_{h}}-(\nabla g, \nabla w)_{\Gamma_{h}} .
\end{aligned}
$$

The proof of (A.15) and hence our theorem will now follow from the following two estimates:

$$
\left|\left(\nabla\left(g_{h}-g\right), \nabla(w-\chi)\right)_{\Omega_{h}}\right| \leq C h d_{j}^{-d / 2}\left\|\nabla\left(g_{h}-g\right)\right\|_{L_{1}\left(\Omega_{h}\right)}+C h\left\|\nabla\left(g_{h}-g\right)\right\|_{\Omega_{j}^{\prime \prime \prime}}
$$

for $\chi$ suitable, and

$$
\left|(\nabla g, \nabla w)_{\Gamma_{h}}\right| \leq C h d_{j}^{-d / 2} .
$$

We begin with (A.17). Since functions in $W_{2}^{2}$ do not necessarily have point values when $d \geq 4$, we will not be able to use the standard interpolant $I_{h} w$ for $\chi$. Instead we take $\chi$ to be a Scott-Zhang type [25] interpolant. The definition and results for this interpolant needed below for curved convex domains are found in [29], Lemma 2.1. We now have

$$
\left|\left(\nabla\left(g_{h}-g\right), \nabla(w-\chi)\right)_{\Omega_{j}^{\prime \prime \prime}}\right| \leq C\left\|\nabla\left(g_{h}-g\right)\right\|_{\Omega_{j}^{\prime \prime \prime}} h\|w\|_{W_{2}^{2}(\Omega)} \leq C h\left\|\nabla\left(g_{h}-g\right)\right\|_{\Omega_{j}^{\prime \prime \prime}},
$$

where we have used the elliptic regularity result $\|w\|_{W_{2}^{2}(\Omega)} \leq C\|\varphi\|=C$.

On the complement of $\Omega_{j}^{\prime \prime \prime}$ we have

$$
\left|\left(\nabla\left(g_{h}-g\right), \nabla(w-\chi)\right)_{\Omega_{h} \backslash \Omega_{j}^{\prime \prime \prime}}\right| \leq C\left\|\nabla\left(g_{h}-g\right)\right\|_{L_{1}\left(\Omega_{h} \backslash \Omega_{j}^{\prime \prime \prime}\right)} h\|w\|_{W_{\infty}^{2}\left(\Omega \backslash A_{j}^{\prime \prime}\right)} .
$$

Since $w(y)=\left(G^{y}, \varphi\right)_{\Omega_{j}^{\prime}}$ for $y \notin \Omega_{j}^{\prime}$, we have, by Lemma A.1 and the Cauchy-Schwarz inequality, for $|\alpha|>0$,

$$
\left|D^{\alpha} w(y)\right| \leq C \operatorname{dist}\left(y, \Omega_{j}^{\prime}\right)^{-d+2-|\alpha|}\|\varphi\|_{L_{1}\left(\Omega_{j}^{\prime}\right)} \leq C \operatorname{dist}\left(y, \Omega_{j}^{\prime}\right)^{-d+2-|\alpha|} d_{j}^{d / 2} .
$$

In particular, $\|w\|_{W_{\infty}^{2}\left(\Omega \backslash A_{j}^{\prime \prime}\right)} \leq C d_{j}^{-d / 2}$, and so

$$
\left|\left(\nabla\left(g_{h}-g\right), \nabla(w-\chi)\right)_{\Omega_{h} \backslash \Omega_{j}^{\prime \prime \prime}}\right| \leq C h d_{j}^{-d / 2}\left\|\nabla\left(g_{h}-g\right)\right\|_{L_{1}\left(\Omega_{h}\right)},
$$

which completes the proof of A.17.

Similarly, for the proof of A.18, we split our considerations over $\Gamma_{h} \cap A_{j}^{\prime \prime}$ and $\Gamma_{h} \backslash A_{j}^{\prime \prime}$, respectively. First, since the volume of $\Gamma_{h} \cap A_{j}^{\prime \prime}$ is bounded by $C h^{2} d_{j}^{d-1}$, 
we have by (A.10) and Sobolev's inequality, with $0<\frac{1}{2}-\frac{1}{p}<\frac{1}{d}$ and $\frac{1}{p}+\frac{1}{q}=1$,

$$
\begin{aligned}
(\nabla g, \nabla w)_{\Gamma_{h} \cap A_{j}^{\prime \prime}} & \leq C d_{j}^{-d}\|\nabla w\|_{L_{1}\left(\Gamma_{h} \cap A_{j}^{\prime \prime}\right)} \leq C d_{j}^{-d}\left(h^{2} d_{j}^{d-1}\right)^{1 / q}\|w\|_{W_{p}^{1}(\Omega)} \\
& \leq C d_{j}^{-d}\left(h^{2} d_{j}^{d-1}\right)^{1 / q}\|w\|_{W_{2}^{2}(\Omega)} \leq C d_{j}^{-d}\left(h^{2} d_{j}^{d-1}\right)^{1 / q} .
\end{aligned}
$$

Since $h \leq C d_{j}$, and choosing $p$ such that $d\left(\frac{1}{2}-\frac{1}{p}\right) \geq \frac{1}{p}$, the right-hand side may be estimated as

$$
C d_{j}^{-d}\left(h^{2} d_{j}^{d-1}\right)^{\frac{1}{q}} \leq C h d_{j}^{-d / 2} d_{j}^{\frac{2}{q}-1-\frac{d}{2}+\frac{d-1}{q}}=C h d_{j}^{-d / 2} d_{j}^{d\left(\frac{1}{2}-\frac{1}{p}\right)-\frac{1}{p}} \leq C h d_{j}^{-d / 2} .
$$

We finally consider the contribution from $\Gamma_{h} \backslash A_{j}^{\prime \prime}$. Here, by (A.10), $|\nabla g(y)| \leq$ $C \rho(y)^{-d}$. Also, since $\operatorname{dist}\left(\Gamma_{h} \backslash A_{j}^{\prime \prime}, \Omega_{j}^{\prime}\right) \geq d_{j}$, we have from A.19 that, in the region under consideration, $|\nabla w(y)| \leq C d_{j}^{-d / 2+1}$, and hence, cf. (A.2),

$$
\left|(\nabla g, \nabla w)_{\Gamma_{h} \backslash A_{j}^{\prime \prime}}\right| \leq C d_{j}^{-d / 2+1} \int_{\Gamma_{h}} \rho(y)^{-d} d y \leq C h d_{j}^{-d / 2+1} \leq C h d_{j}^{-d / 2} .
$$

This completes the proof.

\section{REFERENCES}

[1] S.Agmon, A.Douglis, and L.Nirenberg, Estimates near the boundary for solutions of elliptic partial differential equations satisfying general boundary conditions, I. Comm. Pure Appl. Math. 12 (1959), 623-727. MR 23:A2610

[2] N.Yu.Bakaev, Maximum norm resolvent estimates for elliptic finite element operators, BIT 41 (2001), 215-239. MR 2002c:65197

[3] N.Yu.Bakaev, S.Larsson, and V.Thomée, Long time behavior of backward difference type methods for parabolic equations with memory in Banach space, East-West J. Numer. Math. 6 (1998), 185-206. MR 99j:65143

[4] C.M. Chen and Y.Q. Huang, $W^{1, \infty}$-stability of finite element solutions of elliptic problems, Hunan Annals of Mathematics 6 (1986), 81-89. (In Chinese.) MR 92e:65144

[5] H. Chen, An $L^{2}$ and $L^{\infty}$-Error Analysis for Parabolic Finite Element Equations with Applications by Superconvergence and Error Expansions, Thesis, Heidelberg University 1993.

[6] M.Crouzeix, S.Larsson, and V.Thomée, Resolvent estimates for elliptic finite element operators in one dimension, Math. Comp. 63 (1994), 121-140. MR 95b:65134

[7] M.Crouzeix and V.Thomée, Resolvent estimates in $l_{p}$ for discrete Laplacians on irregular meshes and maximum-norm stability of parabolic finite difference schemes, Comput. Meth. Appl. Math. 1 (2001), 3-17. MR 2002c:65151

[8] J. Descloux, On finite element matrices, SIAM J. Numer. Anal 9 (1972), 260-265. MR 46:8402

[9] G.Da Prato and E.Sinestrari, Differential operators with nondense domain, Ann. Scuola Norm. Sup. Pisa 14 (1987), 285-344. MR 89f:47062

[10] J.Douglas, Jr., T.Dupont, and L.B.Wahlbin, The stability in $L^{q}$ of the $L^{2}$-projection into finite-element function spaces, Numer. Math. 23 (1975), 193-197. MR 52:4669

[11] H.Fujii, Some remarks on finite element analysis of time-dependent field problems, in Theory and Practice in Finite Element Structural Analysis, University of Tokyo Press (1973), 91-106.

[12] R.Haverkamp, Eine Aussage zur $L_{\infty}$-Stabilität und zur genauen Konvergenzordnung der $H_{0}^{1}$-Projektionen. Numer. Math. 44 (1984), 393-405. MR 86f:65194

[13] J.P.Krasovskii, Isolation of singularities of the Green's function, Izv. Akad. Nauk SSSR 31 (1967), 935-966. MR 36:6788

[14] J.A.Nitsche and A.H. Schatz, Interior estimates for Ritz-Galerkin methods, Math. Comp. 28 (1974), 937-958. MR 51:9525

[15] J.A.Nitsche and M.F. Wheeler, $L_{\infty}$-boundedness of the finite element Galerkin operator for parabolic problems, Numer. Funct. Anal. Optimization 4 (1981-82), 325-353. MR 84a:65083

[16] C.Palencia, Maximum norm analysis of completely discrete finite element methods for parabolic problems, SIAM J. Numer. Anal. 33 (1996), 1654-1658. MR 97e:65099 
[17] A.Pazy, Semigroups of Linear Operators and Applications to Partial Differential Equations, Springer-Verlag, New York - Berlin - Heidelberg - Tokyo, 1983. MR 85g:47061

[18] R. Rannacher, $L^{\infty}$-stability estimates and asymptotic error expansion for parabolic finite element equations, in Extrapolation and Defect Correction, Bonner Math. Schriften 228 (1991), 74-94. MR 93i:65093

[19] R.Rannacher and R.Scott, Some optimal error estimates for piecewise linear finite element approximations, Math. Comp. 38 (1982), 437-445. MR 83e:65180

[20] A.H.Schatz, Pointwise error estimates and asymptotic error expansion inequalities for the finite element method on irregular grids. Part1, Global estimates, Math. Comp. 67 (1998), 877-899. MR 98j:65082

[21] A.H.Schatz, V.Thomée, and L.B.Wahlbin, Maximum norm stability and error estimates in parabolic finite element equations, Comm. Pure Appl. Math. 33 (1980), 265-304. MR 81g:65136

[22] A.H.Schatz, V.Thomée, and L.B.Wahlbin, Stability, analyticity, and almost best approximation in maximum norm for parabolic finite element equations, Comm. Pure Appl. Math. 51 (1998), 1349-1385. MR 99h:65171

[23] A.H.Schatz and L.B.Wahlbin, On the quasi-optimality in $L_{\infty}$ of the $\stackrel{o}{H}^{1}$-projection into finite element spaces, Math. Comp. 38 (1982), 1-22. MR 82m:65106

[24] R.Scott, Optimal $L^{\infty}$ estimates for the finite element method on irregular meshes, Math. Comp. 30 (1976), 681-697. MR 55:9560

[25] L.R.Scott and S.Zhang, Finite element interpolation of non-smooth functions satisfying boundary conditions, Math. Comp. 54 (1990), 483-493. MR 90j:65021

[26] H.B.Stewart, Generation of analytic semigroups by strongly elliptic operators, Trans. Amer. Math. Soc. 199 (1974), 141-161. MR 50:10532

[27] V.Thomée, Galerkin Finite Element Methods for Parabolic Problems, Springer Series in Computational Mathematics, Springer - Verlag, Berlin - Heidelberg - New York, 1997. MR 98m:65007.

[28] V.Thomée and L.B.Wahlbin, Maximum-norm stability and error estimates in Galerkin methods for parabolic equations in one space varible, Numer. Math. 41 (1983), 345-371. MR 85f:65099

[29] V.Thomée and L.B.Wahlbin, Stability and analyticity in maximum-norm for simplicial Lagrange finite element semidiscretizations of parabolic equations with Dirichlet boundary conditions, Numer. Math. 87 (2000), 373-389. MR 2001k:65139

[30] H.Triebel, Interpolation Theory, Function Spaces, Differential Operators, VEB Deutscher Verlag, Berlin, 1978. MR 80i:46032a

[31] L.B.Wahlbin, A quasi-optimal estimate in piecewise polynomial Galerkin approximation of parabolic problems, in Numerical Analysis (Dundee, 1981), Lecture Notes in Mathematics No. 912, Springer-Verlag, Berlin-New York, 1982, pp. 230-245. MR 83f:65157

Department of Mathematics, Institute of Economics and Business, Berzarina St. 12, Moscow 123298, Russia

E-mail address: bakaev@postman.ru

Department of Mathematics, Chalmers University of Technology, S-41296 Göteborg, SWEDEN

E-mail address: thomee@math.chalmers.se

Department of mathematics, Cornell University, Ithaca New York 14853

E-mail address: wahlbin@math.cornell.edu 